\title{
Ayudar y documentar durante esta pandemia de COVID-19
}

Estamos viviendo tiempos extraordinarios, no solo para la salud, sino también para la investigación y la práctica de la educación matemática.

Nunca antes se había registrado un éxodo global de profesores y estudiantes de matemáticas, de todos los niveles educativos, hacia escenarios de instrucción basados en el uso intensivo de Internet. Quizá ahora como nunca se requiere de un involucramiento profundo de los padres en la instrucción matemática de sus hijos, debido a la prevalencia de la educación en casa. El escenario pandémico que estamos viviendo ha hecho evidente la importancia de contar con una ciudadanía matemáticamente alfabetizada, capaz de interpretar la información oficial acerca de la emergencia sanitaria.

Nunca en la historia de la investigación en educación matemática se había dado un aplazamiento en cadena de la mayoría de los congresos internacionales y regionales de nuestra disciplina. Los tiempos de revisión y de procesamiento de artículos de la mayoría de las revistas de investigación se han visto afectados, en gran medida, porque las circunstancias y la capacidad de trabajo de los académicos que revisan y editan los manuscritos ha sido afectada por la pandemia. Muchos proyectos de investigación han sido interrumpidos o rediseñados, dada la repentina interrupción en el acceso a los datos y a los escenarios de experimentación empírica originalmente planificados. 
Considero que es parte de nuestra responsabilidad documentar las perturbaciones que esta pandemia de COVID-19 está causando en las diferentes esferas de la educación matemática, además de apoyar a las personas afectadas por tales perturbaciones.

Parte de esta responsabilidad es con la historia. Imaginemos por un momento a las comunidades futuras de educadoras y educadores matemáticos dentro de, digamos, quince o veinte años. Pensemos que recurren a los anales de la educación matemática tratando de entender cómo en el pasado la pandemia de COVID-19 afectó a la instrucción matemática en Iberoamérica. Supongamos ahora que después de su búsqueda no encuentran nada. Ni un estudio, ni un reporte, ni un testimonio. Como si nada hubiera sucedido. Pienso que sería una gran omisión por parte nuestra como educadores matemáticos.

Sin embargo, la responsabilidad mayor es con la sociedad en general. Hoy profesores, estudiantes, padres de familia y administrativos requieren de consejo y respuestas por parte de los especialistas en la educación matemática: ¿cómo enseñar matemáticas y mantener motivados a los estudiantes a través de videoconferencia?, ¿qué prácticas y condiciones podrían favorecer el aprendizaje de las matemáticas en un escenario de instrucción a distancia como el que vivimos?, ¿cómo atender en este momento la educación matemática de poblaciones alejadas o marginadas con acceso limitado o nulo a las tecnologías digitales?, ¿cómo fortalecer y promover el conocimiento matemático para el involucramiento de los padres en la educación matemática de los niños desde casa?, ¿qué competencias matemáticas se pueden promover entre la ciudadanía para prepararla a interpretar información en escenarios de emergencia sanitaria?

Debemos contribuir a responder este tipo de preguntas sintetizando investigación y resultados ya existentes, y que abordan problemáticas relacionadas con temas como: la educación matemática en línea, el involucramiento de los padres en el desarrollo matemático de los hijos, la alfabetización matemática, los procesos de integración de tecnología en la práctica docente, entre otros. Se podría también contribuir estudiando las nuevas prácticas y problemáticas de la instrucción matemática, y dando voz a los distintos actores involucrados en ellas. Incluso sería posible apoyar organizando y promoviendo oportunidades de desarrollo especializado para estudiantes, padres de familia y docentes. Hay mucho por hacer, estudiar y documentar.

Los invito a no perder la oportunidad que esta crisis nos da para contribuir y ayudar a otros desde nuestra posición como educadoras y educadores 
matemáticos. Pero también, les invito a documentar sus esfuerzos y sus estudios, para posteriormente publicarlos y divulgarlos. Como lector estaría entusiasmado de presenciar un creciente flujo de publicaciones y estudios de buena calidad provenientes de Iberoamérica, que aborden aspectos de la relación entre la educación matemática y la pandemia de COVID-19. Como editor asociado me complacería que varios de esos estudios estuvieran publicados en la revista Educación Matemática.

Mario Sánchez Aguilar Editor Asociado 